\title{
A second-generation motor rhythm analysis system
}

\author{
FRANK L. SMOLL and RONALD A. PALMATIER \\ Department of Physical Education, University of Washington, Seattle, Washington 98195
}

\begin{abstract}
This report describes the design and operation of a second-generation motor rhythm analysis system (MRAS). The system enables quantification of spatial and temporal characteristics of movements performed in synchronization with audio-visual rhy thmic stimuli. The MRAS also provides for assessment of preferred tempo in performance of repetitive movements. In addition to discussing the rationale for developing the system, consideration is given to procedures for its utilization in research regarding motor rhythm and preferred tempo.
\end{abstract}

Rhythm is generally regarded as a basic component underlying the acquisition and performance of motor skills. Consequently, numerous studies have focused on performers' abilities to match their motor responses to the rhythmic structure of imposed stimulus series. Unfortunately, earlier investigations of motor rhythm shared a procedural shortcoming in that they were concerned with assessment of only temporal precision. Recognizing that movement is a space-time organization of events, a motor rhythm analysis system (MRAS) was designed and contructed (Smoll, 1973) with the capacity for quantification of space-time characteristics of movements performed in synchronization with auditory rhythmic stimuli-a system enabling assessment of the ability to be in a specific point in space (spatial accuracy) at a specific point in time (temporal accuracy). With the MRAS, the motor response is an underarm swing comparable to the fundamental motor pattern commonly found in performance of numerous motor skills. We have used the MRAS extensively (Smoll, 1974a, 1974b, 1975a), and it has served as a prototype for instrumentation in motor rhythm research of others (Thomas \& Halliwell, 1976; Thomas \& Moon, 1976). The system has also been incorporated into the study of repetitive movements at self-paced tempos, that is, the preferred tempo phenomenon (Smoll, 1975b, 1975c, 1975d). Although data collection procedures are well established, quantification of the original MRAS analog proved to be laborious and extremely time consuming. The second-generation MRAS was designed to overcome this problem.

\section{DESCRIPTION OF THE MRAS}

The MRAS (Figure 1) consists of an electronic metronome, a target, and electromechanical components that transmit voltage fluctuations representing elevationdepression arm movements in the sagittal plane to electronic processing circuitry, and on to a digital printer. The support stand is made of two telescoping pipes, with a setscrew for height adjustment. The larger pipe is affixed to a steel base, which is bolted to a plywood platform for stability. A steel shaft is mounted in a bearing assembly, which is affixed perpendicular to the upper end of the support stand. A potentiometer transducer is secured coaxially to the shaft and wired to the processing circuitry.

A swing bar consisting of two telescoping aluminum tubes is attached to the end of the shaft; a compression lock enables adjustment of the bar length. A freely rotating handle is located $7.6 \mathrm{~cm}$ from the distal end of the bar, perpendicular to the bar and parallel to the shaft. An L-shaped brass sighting rod, $.3 \mathrm{~cm}$ in diam, is attached at the distal end of the bar. The $5.1-\mathrm{cm}$ segment of the rod is orthogonal with the bar and the handle; and the 10.2-cm segment, which is painted black, extends parallel to the handle. A flexible piece of plastic is clamped perpendicular to the support stand

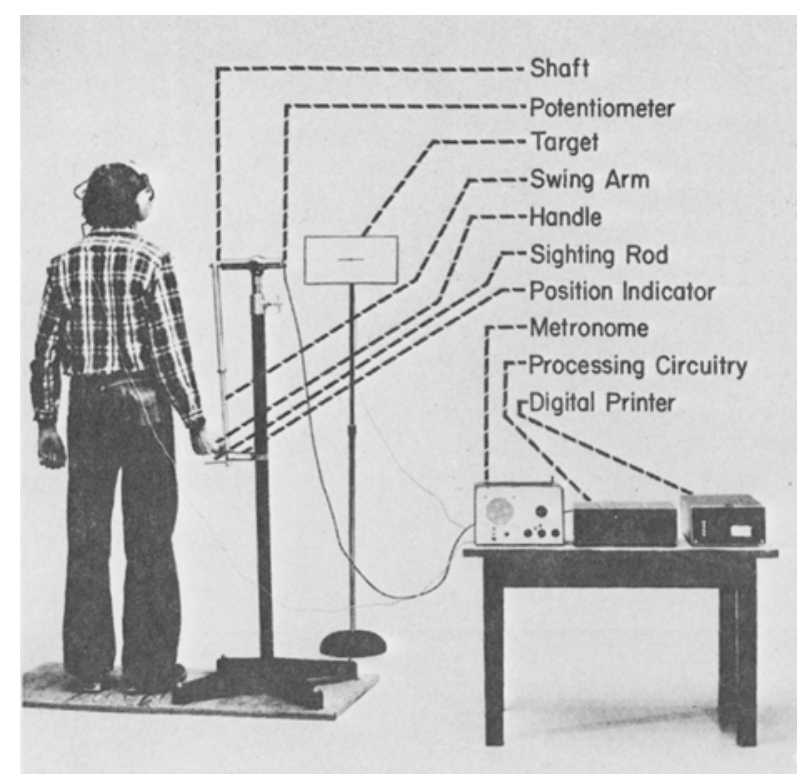

Figure 1. Motor thythm analysis system. 
to serve as a vertical position indicator for the subject.

The target is a black horizontal line, $10.2 \mathrm{~cm}$ long and $.3 \mathrm{~cm}$ wide, centered on a white Masonite background. The target is fastened to another support stand made of two telescoping pipes secured to a steel base. A compression lock enables adjustment of the target height.

The electronic metronome (FKS Timing Systems Model 200) emits auditory stimuli at time intervals selectable at .1-sec gradations. The audio "beeps" are presented to the subject via a headset. The metronome has another selectable feature; on alternate beats, it activates a red light-emitting diode, $.3 \mathrm{~cm}$ in diam, which is centered on the target line.

In addition to the potentiometer and metronome, the electronic portion of the system (see Figure 2) includes custom processing circuitry, a digital panel meter (Newport Model 200), a timer (Newport Model 61 10), and a digital printer (Newport Model 810).

\section{EXPERIMENTAL PROCEDURES AND MRAS OPERATION}

While the subject assumes a normal standing position with his side to the swing bar, the height of the shaft is adjusted to correspond to the axis of rotation of his humerus at the glenohumeral joint. The bar length is then adjusted, enabling the subject to grasp the handle in a supinated position. With the bar placed horizontally, the target height is adjusted to align the target line and the sighting rod. The target surface is positioned close to the sighting rod to preclude parallax error. The position indicator is adjusted to contact the swing bar when it is vertical.

Two modes of MRAS operation have been established. When obtaining measures of spatial and temporal elements of motor rhythm (Mode A), the metronome is set to emit audio stimuli at a specified time interval (tempo) and to activate the light-emitting diode on

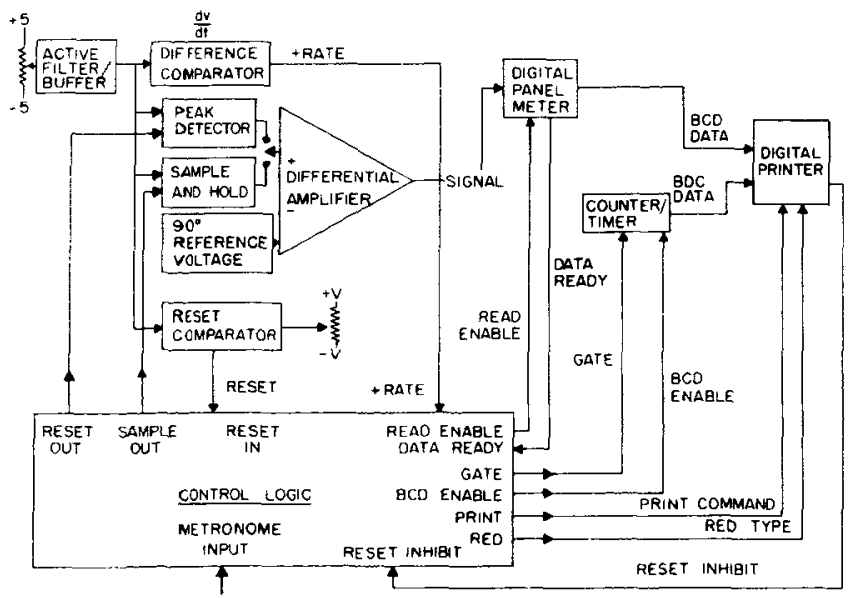

Figure 2. Functional block diagram of the MRAS. alternate beats. The subject's task is to swing his arm from a vertical starting position forward and upward in a continuous motion, bringing the sighting rod in line with the target line. The subject attempts to "hit" the target line at a time coincident with the audio-visual stimulus (simultaneous beep and flash of light) and returns to the starting position on the subsequent audio stimulus. The measure of spatial accuracy is the deviation between the sighting rod and the target line at the instant the audio-visual stimulus occurs, which is reflected by the degrees the swing bar deviates from the horizontal reference. Temporal accuracy is the time difference between the occurrence of the apex of the oscillation and the audio-visual stimulus.

The potentiometer has $\pm 5-\mathrm{V}$ dc excitation, enabling it to transduce oscillating motion of the swing bar to a varying voltage in proportion to the amount of bar displacement. This signal is applied to a sample-and-hold network to determine the amplitude of the swing at the time at which the audio-visual stimulus occurs. The voltage representing angular displacement is then compared to a reference voltage equivalent to $90 \mathrm{deg}$. The difference voltage from this comparison is applied to the digital panel meter for analog-to-digital conversion and display, and thence to the digital printer for a record of the amplitude difference (spatial accuracy) in degrees to the nearest tenth. If the amplitude at metronome time exceeds the reference, the polarity is positive; if it is less than the reference, the polarity is negative.

Meanwhile, the temporal peak pulse is logically "ORed" to the metronome pulse, yielding an interval which gates the counter for a time equal to the difference in milliseconds between the two pulses. This time interval (temporal accuracy) is displayed and applied to the digital printer for simultaneous printout with the associated spatial accuracy value. If the time of the peak is prior to or coincident with the metronome pulse, the digital printout is black; if subsequent to the metronome pulse, the printout is red.

When obtaining measures of preferred tempo (Mode B), neither audio or visual stimuli are incorporated. The subject's task is to swing his arm from a vertical starting position forward and upward in a continuous motion until the sighting rod is in line with the target line, and then return to the starting position. The subject repeats the movements at a self-chosen tempo that is "most comfortable" for him-a tempo that is "most convenient and natural." In Mode B, the object is to measure the time for each complete movement cycle (i.e., period between the apexes of the upward arm swings). The counter is gated on by the first peak and off by the next peak; and after a delay of less than $1 \mathrm{msec}$, the sequence is repeated. The time in milliseconds is displayed and output on the digital printer. 


\section{CONCLUDING STATEMENT}

Although preferred tempo is believed to be a potentially influential factor for a variety of tasks, relatively little information is available concerning its role in skill acquisition and performance. The secondgeneration MRAS provides instrumentation for future research designed to elucidate the relationship between preferred tempo and motor rhythm, and thus enhance our understanding of rhythmic motor behavior.

\section{REFERENCES}

Smoll, F. L. A rhythmic ability analysis system. Research Quarterly, 1973, 44, 232-236.

Smolt, F. L. Development of spatial and temporal elements of rhythmic ability. Journal of Motor Behavior, 1974, 6. 53-58. (a)

Smols, F. L. Development of rhythmic ability in response to selected tempos. Perceptual and Motor Skils, 1974, 39, 767-772. (b)
SMoll, F. L. Variability in development of spatial and temporal elements of rhythmic ability. Perceptual and Motor Skills, 1975. 40. 140. (a)

Smoll, F. L. Preferred tempo in performance of repetitive movements. Perceptual and Motor Skills, 1975, 40, 439-442. (b)

SMOLL, F. L. Preferred tempo of motor performance: Individual differences in within-individual variability. Journal of Motor Behavior, 1975. 7. 259-263. (c)

Smoll, F. L. Between-days consistency in personal tempo. Perceptual and Motor Skills, 1975, 41, 731-734. (d)

Thomas, J. R., \& Halliwell, W. Individual differences in motor skill acquisition. Journal of Motor Behavior, 1976 , 8. 89-99.

Thomas, J. R., \& Moon, D. H. Measuring motor rhythmic ability in children. Research Quarterly, 1976, 47, 20-32.

(Received for publication May 13, 1977; revision accepted June 29,1977 .) 